TITLE:

\title{
DNA nanotechnology: Measuring chloride in live cells
}

AUTHOR(S):

Endo, Masayuki; Sugiyama, Hiroshi

CITATION:

Endo, Masayuki ...[et al]. DNA nanotechnology: Measuring chloride in live cells. Nature Nanotechnology 2015, 10(7): 569-570

ISSUE DATE:

2015-07-03

URL:

http://hdl.handle.net/2433/230938

RIGHT:

This is the accepted manuscript of the article, which has been published in final form at

https://www.nature.com/articles/nnano.2015.142.; The full-text file will be made open to the public on 03 January 2016 in accordance with publisher's 'Terms and Conditions for Self-Archiving'.; この論文は出版社版でありません。引用の際 には出版社版をご確認ご利用ください。; This is not the published version. Please cite only the published version. 


\section{Chloride Sensor: measuring sub-cellular chloride in live cells}

A nucleic acid based chloride sensor Clensor images and quantifies spatiotemporal chloride transport associated with endosomal maturation in the living cell.

Masayuki Endo and Hiroshi Sugiyama

Quantitative molecular imaging is a rapidly growing technology for quantification of chemicals and biological molecules and for monitoring phenomena in the living cell. Chloride is one of the target chemicals for intracellular sensing, which functions for $\mathrm{pH}$ regulation, cell volume regulation, cell migration, and cell proliferation. In the cytosol and lumen of organelles, chloride concentration is stringently regulated by the chloride transporters and channels. ${ }^{1,2}$ Despite the importance of monitoring chloride in the organelles, the real time quantification in a specific organelle using a fluorescent probe is still challenging because it requires the ability to monitor a wide range of chloride concentration and should also be insensitive to $\mathrm{pH}$ changes. Writing in Nature Nanotechnology, Yamuna Krishnan and colleagues report a nucleic acid based chloride sensor called "Clensor", which monitors spatiotemporal chloride concentration in the organelles in the living cell. The problem of the present protein-based chloride sensors is their pH sensitivity and their restriction to specific, narrow ranges of $\mathrm{Cl}^{-}$concentration. ${ }^{3}$

Krishnan and colleagues overcome these limitations using functionalized nucleotides that contain sensing, normalizing, and targeting modules (Figure 1a). Nucleic acid-based sensors are widely investigated for investigating various phenomena in living cells especially by fluorescence imaging.,5 They employed a fluorescent and chloride-sensitive molecule, 10,10'-Bis[3- carboxypropyl]-9,9'-biacridinium dinitrate (BAC), which was used for sensing 
chloride in subcellular compartments. ${ }^{6,7}$ The chloride insensitive Alexa 647 fluorophore was used for normalizing the fluorescence intensity in a ratiometric fashion. By simply assembling these modules, the quantitative chloride reporting nanodevice Clensor was realised (Figure 1a). The $\mathrm{R} / \mathrm{G}$ ratio of Clensor, where the fluorescence intensity of Alexa $647(\mathrm{R}$ ) is divided by that of BAC (G), shows a linear dependence on $\mathrm{Cl}^{-}$concentration ranging from $5 \mathrm{mM}$ to $200 \mathrm{mM}$ and is also insensitive to solution pH (Figure $1 \mathrm{~b}$ ). Clensor shows excellent $\mathrm{Cl}^{-}$sensing performance in vitro experiments, as well as quantitatively monitors intracellular $\mathrm{Cl}^{-}$concentration (Figure 1b).

Krishnan and colleagues applied Clensor to determine the $\mathrm{Cl}^{-}$concentration in specific endo-lysosomal compartments including early endosome (EE), late endosome (EE), lysosome (LY), and recycling endosome (RE). Clensor is internalized by endocytosis via anionic ligand binding receptor (ALBR) pathway. Then endosomal/lysosomal maturation was monitored by R/G ratio imaging with Clensor having confirmed organelle localization using specific endocytic markers. Remarkably, Clensor can acquire organelle specific targeting ability by just replacing the targeting module of the nanodevice. Conjugating an RNA aptamer targeting a transferrin receptor, Clensor can be internalized via transferrin pathway (Clensor ${ }^{T f}$ ), which was efficiently uptaken by transferrin receptor expressing cells.

Intracellular functionality of Clensor was examined by $\mathrm{R} / \mathrm{G}$ ratio by comparing experiments in vitro and in the endosome of Drosophila hemocytes by changing $\mathrm{Cl}^{-}$concentrations. In both experiments, $\mathrm{R} / \mathrm{G}$ ratios show the good agreements of the quantitative performance of Clensor in a range of 5-120 $\mathrm{mM}$ (Figure $1 \mathrm{c})$.

Next, Krishnan and colleagues demonstrated the quantification of $\mathrm{Cl}^{-}$concentrations during the endosome-lysosome maturation. $\mathrm{Cl}^{-}$concentrations in endosome and lysosome were directly measured by R/G ratio with Clensor. Compartments are acidified along the endocytic pathway from $\mathrm{pH} 6.0$ in early endosomes to $\mathrm{pH} \sim 5.0$ in lysosomes, accompanying the increase of $\mathrm{Cl}^{-}$ 
concentration. From the distribution of $\mathrm{R} / \mathrm{G}$ ratios, $\mathrm{Cl}^{-}$concentration increased during the endosome-lysosome maturation. Importantly, $\mathrm{Cl}^{-}$concentration in lysosomes was first measured using this sensor, whose values are consistent with the theoretical model.

Finally, they demonstrate characterization of the functions of the putative CLC family proteins, DmClC-b and DmClC-c, during the endosome-lysosome maturation. To investigate the function of DmClC-b and DmClC-c, the expression of these proteins was suppressed by the corresponding RNAi and the R/G ratios were measured in each endocytic organelle and also correlated to lumenal $\mathrm{pH}$. They found that $\mathrm{DmClC}-\mathrm{c}$ accumulates $\mathrm{Cl}^{-}$in the early endosome with acidification. On the other hand, DmClC-c worked in late endosome and lysosome to accumulate $\mathrm{Cl}^{-}$, especially in the lysosome, without acidification. Clensor clearly revealed the different roles of these proteins in endolysosomal maturation.

Krishnan and colleagues successfully created a structurally simple nucleic acid based nanodevice by combining the functionalized modules to integrate the sensing and targeting functions. These modules can be easily replaced in a "mix-and-match" format for the desired sensing and targeting purposes. Clensor works in subcellular compartments and the sensing ability was completely preserved. This simple nucleic acid-based nanodevice can be a general tool for imaging complicated intracellular phenomena and real time quantification of chloride in the living cell for diverse biological problems.

Masayuki Endo and Hiroshi Sugiyama are in the Institute for Integrated Cell Material Sciences, Kyoto University, Yoshida-ushinomiyacho, Sakyo-ku, Kyoto 606-8501, Japan.

e-mail: endo@kuchem.kyoto-u.ac.jp (ME)and hs@kuchem.kyoto-u.ac.jp (HS). 
References

1. Blaesse, P., Airaksinen, M. S., Rivera, C. \& Kaila, K. Neuron 61, 820-838 (2009).

2. Bregestovski, P., Waseem, T. \& Mukhtarov, M. Front. Mol. Neurosci. 2, 15 (2009).

3. Arosio, D. et al. Nat. Methods 7, 516-518 (2010).

4. Krishnan, Y. \& Bathe M. Trends in Cell Biology. 22, 624-633 (2012).

5. Krishnan, Y. \& Simmel, F. C. Angew. Chem. Int. Ed. 50, 3124-3156 (2011).

6. Sonawane, N. D., Thiagarajah, J. R. \& Verkman, A. S. J. Biol. Chem. 277, 5506-5513 (2002).

7. Biwersi, J., Tulk, B. \& Verkman, A. S. Anal. Biochem. 219, 139-143 (1994).

Figure 1: Clensor functions in the subcellular components.

a

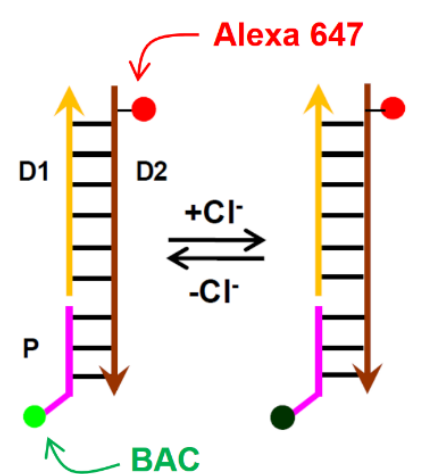

b

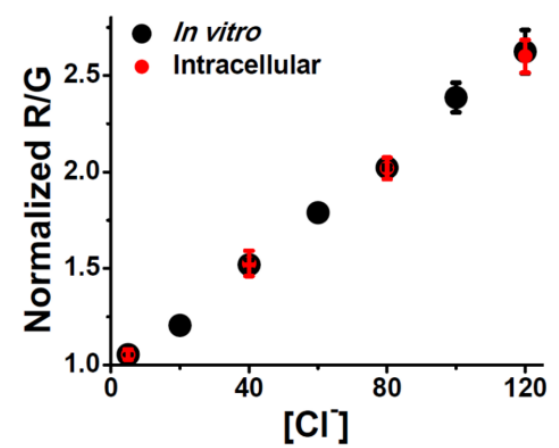

Tf aptamer

C

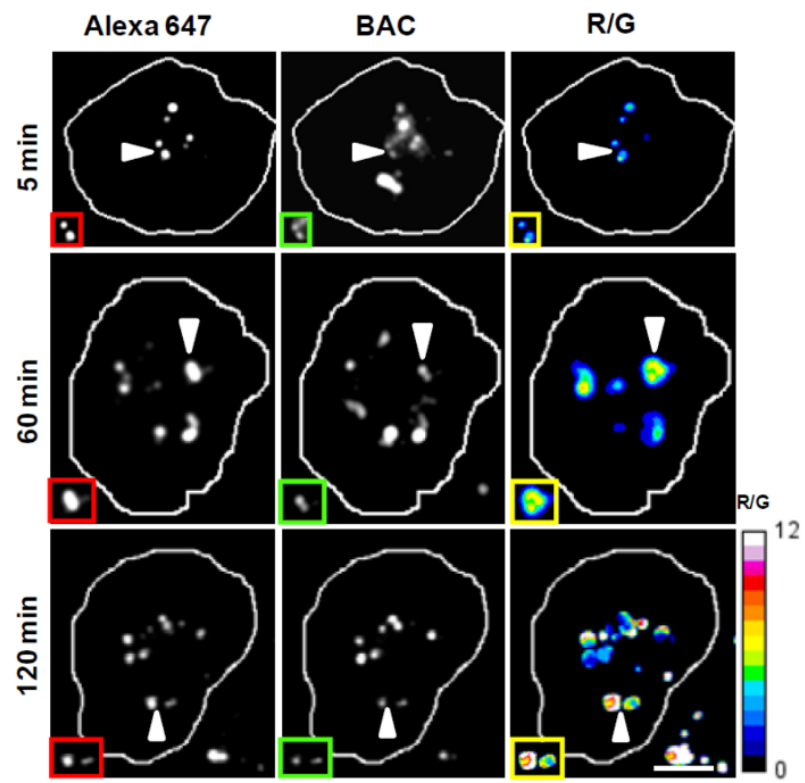


(a) $\mathrm{Cl}^{-}$-sensitive fluorophore BAC (G) and reference Alexa $647(\mathrm{R})$ modules are assembled using DNA and analogs to construct Clensor. Fluorescence of BAC is quenched by increasing of $\mathrm{Cl}^{-}$ concentration. (b) Quantitative performance of Clensor. Linearity of R/G ratios vs. $\mathrm{Cl}^{-}$concentration in vitro and in cell. (c) Alexa 647, BAC, and pseudocolour R/G map of live Drosophila hemocytes labeled with Clensor in EE (5 min), in LE (60 min), and LY (120 min). Scale bar: $10 \mu \mathrm{m}$. 